\title{
On the influence of surface roughness on real area of contact in normal, dry, friction free, rough contact by using a neural network
}

\author{
M. Rapetto ${ }^{1}$, R. Larsson ${ }^{1}$, A. Almqvist ${ }^{1}$, P.M. Lugt ${ }^{1,2}$ \\ ${ }^{1}$ Department of Mechanical Engineering, Division of Machine Elements \\ Luleå University of Technology, Luleå, SE-97187 Sweden \\ ${ }^{2} \mathrm{SKF}$ Engineering and Research Centre \\ P.O.Box 2350, 3430 DT Nieuwegein \\ marco.rapetto@ltu.se
}

\begin{abstract}
A model previously developed at LTU was used in order to perform numerical simulations of normal, dry, friction free, linear elastic contact of rough surfaces. A variational approach was followed and the FFT-technique was used to speed up the numerical solution process.

Five different steel surfaces were measured using a Wyko optical profilometer and several 2-D profiles were taken. The real area of contact and the pressure distribution over the contact length were calculated for all the 2-D profiles. A new slope parameter was defined.

An artificial neural network (ANN) was applied to determine the relationship between the roughness parameters and the real area of con-
\end{abstract}

tact. The roughness parameters were the inputs for the neural network, the target was the real area of contact computed through the model. An ANN model was built using a feedforward neural network made of 2 hidden layers. In parallel another neural network was trained, considering standard parameters and the new roughness parameter. The trained model was able to capture the dependence of the real area of contact on the roughness parameters. The ability of the neural network to generalize on unseen data was tested. The neural network was able to prove the correlation between the roughness parameters and the real area of contact. 